\title{
Compact and Temperature Independent Electro-optic Switch Based on Slotted Silicon Photonic Crystal Directional Coupler
}

\author{
Hassan Aghababaeian and Mohammad-Hashem Vadjed-Samiei* \\ School of Electrical Engineering, Iran University of Science and Technology, Tehran-1684613114, Iran
}

(Received May 31, 2012 : revised July 31, 2012 : accepted August 6, 2012)

\begin{abstract}
In this paper, we have proposed a principle to design a compact and temperature independent electro-optic switch based on a slotted photonic crystal directional coupler (SPCDC). Infiltration of the slotted silicon photonic crystal with polymer enhances the slow light and decreases the switching length, whereas the different signs of thermo-optic coefficients of the polymer and silicon make the proposed switch stable within $25^{\circ} \mathrm{C}$ to $85^{\circ} \mathrm{C}$ temperature range. The SPCDC structure is modified to increase poling efficiency of the polymer in the slot and to flatten the dispersion diagram of the even mode to minimize the switching length.
\end{abstract}

Keywords: Optical switches, Electro-optic devices, Slow light, Photonic crystal, Thermal effects

OCIS codes : (130.3120) Integrated optics devices; (120.6810) Thermal effects; (230.2090) Electro-optical devices; (060.1810) Buffers, couplers, routers, switches, and multiplexers

\section{INTRODUCTION}

Optical switches are essential components in high speed optical communication systems and networks. Implementation of optical switches based on the silicon on insulator (SOI) has attracted many researchers in recent years [1-4]. SOI substrates are intrinsically compatible with CMOS electronics, allowing access to a mature manufacturing infrastructure and the ability to combine photonic and electronic networks on a single chip [5]. The centrosymmetric crystal structure of silicon prohibits native silicon from possessing the Pockels effect, with the result that switches tend to be either very long or require high powers to operate. This problem limits the use of silicon in photonic integrated circuits (PICs) [6]. The thermo-optic effect in silicon is comparatively higher than EO effect, but the switching time above $1 \mu \mathrm{s}$ is an important limitation in thermo-optic devices [2,8].

Slow light (SL) can reduce the power or the physical length of the switch by enhancing the lightwave-matter interaction [7, 9-11]. In the slow light regime, the optical signal is compressed and hence the pulse amplitude is increased, which causes the enhancement of the linear and nonlinear effects in the structure. Linear effects such as gain, thermo- optic and electro-optic are scaled with the slowdown factor, whereas the nonlinear effects scale with its square $[12,13]$.

Photonic crystals (PCs) can be engineered to create new functionalities such as variation of the dispersion diagram to slow down the speed of light [1, 7, 9 and 10]. It is possible to design slow-light photonic crystal structures to attain high slow down factor and low group velocity dispersion (GVD) through engineering the geometry of the photonic crystal structure $[9,10,14,15]$. Injecting the electro-optic (E-O) or nonlinear materials to the slot region of the slotted PCW results in slow down and confinement of the light and tuneability of the devices $[5,16,17$, and 19]. Nevertheless, the in-slot E-O efficiency is relatively low $(\sim 10 \%-30 \%)$ compared with the value of bulk E-O polymer $[20,28]$. Recent research has shown that large leakage current during the poling process can degrade the poling efficiency and E-O coefficient [18]. Using new E-O polymer materials (such as $15 \%$ wt. AJLZ53/polymethyl methacrylate) $[20,21]$ with lower conductivity in a wide slot will significantly reduce the leakage current, and it can increase the thin film poling efficiency [21, 22]. Recently, effective in-device E-O coefficient of $735 \mathrm{pm} / \mathrm{V}$ on electro-optic polymer infiltrated silicon photonic crystal

\footnotetext{
*Corresponding author: mh_samiei@iust.ac.ir

Color versions of one or more of the figures in this paper are available online.
} 
slot waveguides has been demonstrated [21].

Another common problem in silicon photonic devices is the temperature dependence of the optical properties due to the thermo-optic effect of silicon [21]. Although the thermal expansion effect has been considered in some research on optical sensors [38] but variation of the silicon refractive index with temperature has not been taken into account in the previous research on PC switches and modulators, whereas it has a considerable effect on the optical properties of the devices. As an example a $38^{\circ} \mathrm{C}$ temperature variation corresponds to variation of $6.8 \times 10^{-3}$ in refractive index which is enough to perform switching in some PC switches [24, 25]. Hence, reduction of the temperature sensitivity of the device is necessary $[1,26]$.

We present a solution to improve the E-O performance in SOI by means of polymer infiltrated SPCDC to design a slow light photonic crystal switch. As the polymers offer very high Pockels coefficients [5], infiltration of the SPCDC by polymer enhances the E-O effect in the designed structure and causes switching in the very short length. Proper E-O material with low conductivity in a wide slot will sufficiently increase the poling efficiency and enhances in-device E-O coefficient [21, 28, and 29]. However, the thermal dependence of silicon's and polymer's refractive indexes are typically of different sign. So opposing the thermo-optic effect of the polymer can minimize or even eliminate the temperature dependence of the device for a given operating point $[27,30]$. We have shown that the designed structure is stable for temperature variation of $\Delta T=60^{\circ} \mathrm{C}$.

This paper is organized as follows. The design of the slow light slotted PC directional coupler will be discussed in the next Section. The switch is designed and its performance and thermal stability are demonstrated in Sections 3 and 4, respectively. The paper is concluded in Section 5 .

\section{DISPERSION ENGINEERING}

The planar slotted photonic crystal directional coupler can be implemented by replacing two rows of air holes in the triangular lattice $\mathrm{PC}$ with narrow slots, as displayed in FIG. 1. Two slotted photonic crystal waveguides (SPCW) are placed sufficiently close that the optical modes in each waveguide overlap and interact with each other. As for the conventional directional coupler, odd and even supermodes are supported in this structure [1]. Each mode has a different wavevector and therefore light in the system will couple from one waveguide to the other after encurring a relative phase difference of $\pi$. This gives a switching length of $L$ $=\pi / \Delta k$. A large $\Delta k$ is desirable to keep the switching length small. The change of the refractive index of $\Delta n$ can cause a wavevector increase of $\Delta k$ to switch the light from bar to cross port of the device. But the possible change in refractive index of silicon is small and needs a long switching length. Meanwhile $d \omega / d k$ is small in the slow light regime, hence, the slow light enhancement via

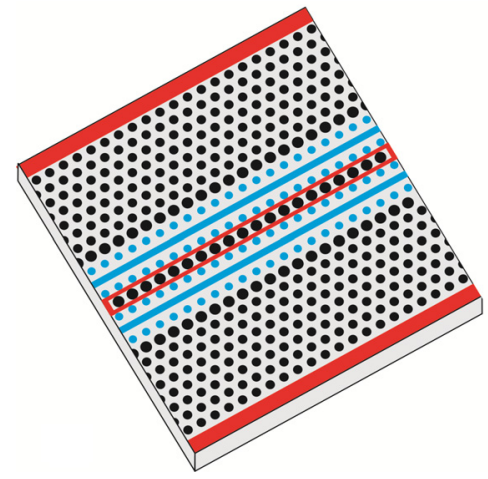

(a)

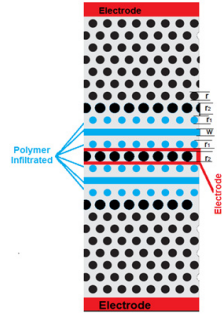

(b)
FIG. 1. (a) 3D geometry of the electro-optic slotted photonic crystal switch implemented on SOI. The slots and the innermost rows of holes are infiltrated with polymer and the radii of the innermost rows of the holes are modified. (b) top view of the device introducing the structure parameters.

dispersion engineering is necessary to shorten the switching length $[1,2]$.

Since the innermost rows of the holes in SPCW have the most important impact on the dispersion diagram [1, $15,31]$, these rows are modified for engineering the dispersion diagram of the odd and even modes. The slot and the innermost rows in each SPCW are infiltrated with polymer, as illustrated in FIG. 1b. The structure is considered to have the geometry parameters of silicon refractive index of $n_{s i}=3.46$ at the wavelength of $1.55 \mu \mathrm{m}$, lattice constant of $a=390 \mathrm{~nm}$ and holes radii of $\mathrm{r}=0.3 a$.

To increase the polling efficiency and enhance the effective E-O coefficient $\left(\mathrm{r}_{33}\right)$, it is necessary to use a wide slot $[21,28]$. Although the effective in-device $r_{33}$ of 735 $\mathrm{pm} / \mathrm{V}$ from an electro-optic polymer infiltrated slotted silicon photonic crystal waveguide with the slot width of $\mathrm{W}=0.75 a$ has been demonstrated [21], but our simulation shows the slots wider than $0.6 a$ shifts the slow light to near band edge and increase the GVD of the structure of FIG. 1. Hence, the slot width of $\mathrm{W}=0.55 a$ is considered for our design to be wide enough to enhance E-O coefficient and prevent high GVD. In this work, we propose to use $25 \%$ wt. AJCKL1/amorphous polycarbonate low-loss and low birefringent polymers as infiltrating materials [21]. Its refractive index is about 1.63 at wavelength of $1.55 \mu \mathrm{m}$ [21]. Two electrodes will be deposited longitudinally alongside the device to apply voltage and another electrode on the top of the innermost row of the holes to be grounded as depicted in FIG. 1.

Figure 2(a) shows the odd and even supermodes in the band gap. The flat band part of the even mode and operation point of the switch is below the light line and it means a good light confinement in the vertical direction of the slab $[9,39]$, hence two dimensional simulation is precise enough to estimate the dispersion diagrams of the proposed structure [39]. Two dimensional numerical calculations of the band diagrams are performed by the plane wave expansion method (PWEM) and by selecting an appropriate supercell using 


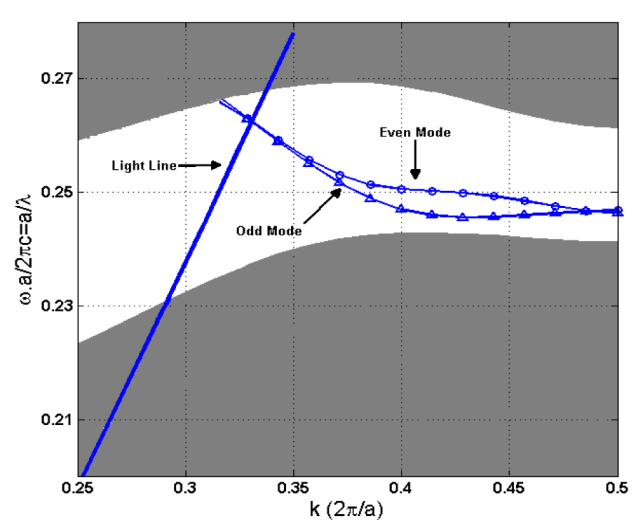

(a)

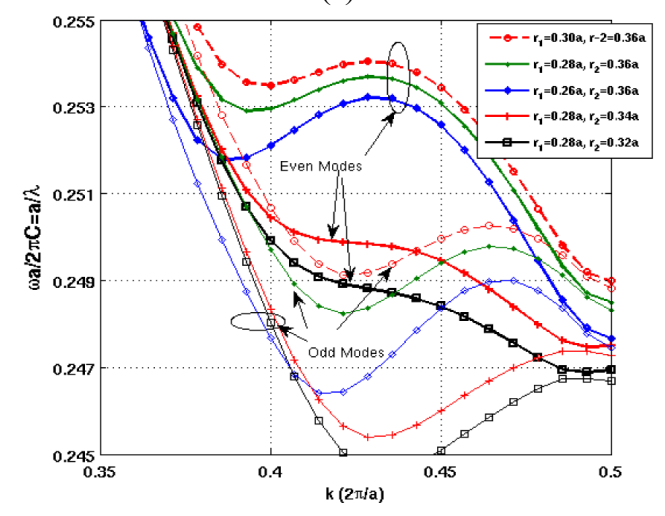

(b)

FIG. 2. (a) Calculated dispersion diagram for $\mathrm{r}_{1}=0.28 a$ and $\mathrm{r}_{2}$ $=0.34 a$ and $(b)$ dependence of the dispersion diagram on the radius of the first two rows of the holes, $r_{1}$ and $r_{2}$. The higher $r_{2}$ will increase the tail of the diagram near band edge while higher $\mathrm{r}_{1}$, will increase the diagram at the middle of the band.

the MIT photonic band (MPB) package [32]. We can see the dependence of the dispersion diagram to the diameter of the first two rows of the holes in FIG. 2(b). It shows that the increment in radius of second row of holes, $r_{2}$, will increase the tail of the diagram near band edge while increment in radius of first rows, $r_{1}$, will increase the diagram at the middle of the band. The optimum values $r_{1}$ $=0.28 a$ and $\mathrm{r}_{2}=0.34 a$ will maximize the slow light effect and flatten the band diagram of the even mode. Utilizing the optimum values of $r_{1}, r_{2}$ and other above mentioned device parameters minimize the value of $d \omega / d k$. Therefore a small vertical shift in the band diagram causes a large change on $\Delta k$ in desired frequency and enables switching in a short length. The required vertical shift in the band diagram can be implemented by a small change in the polymer's refractive index. The switching mechanism and switch design will be discussed in detail in the next section.

\section{SWITCH DESIGN}

Applying electrical voltage to the electrodes of the

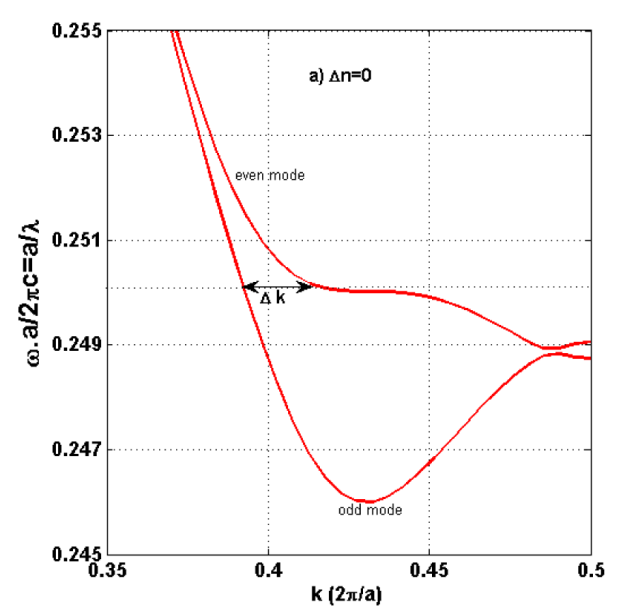

(a)

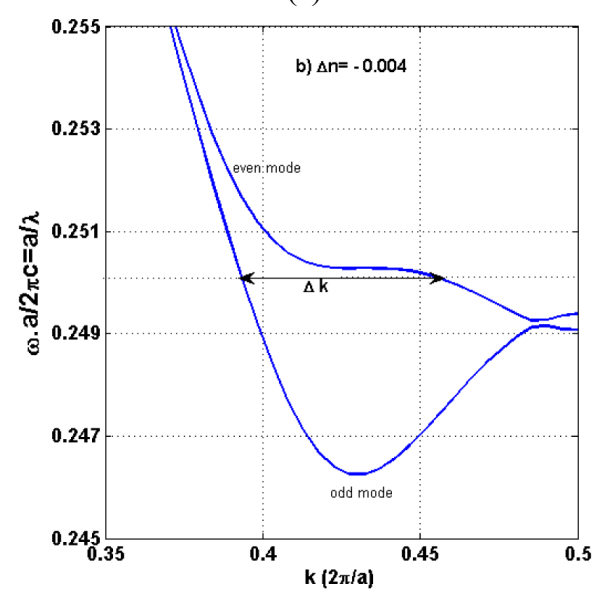

(b)

FIG. 3. Dispersion diagram for even and odd modes with a shift in the refractive index of polymer. A shift in refractive index causes a large change in even mode's and a very small change in odd mode's wavevector at the normalized frequency of $a / \lambda=0.250$.

structure of FIG. 1 changes the refractive index of polymer at the slots. To apply electrical field directly to the slot, the dopant concentration of $n_{D}=10^{17}$ is necessary $[5,16]$. Although an additional optical loss of $\approx 2 \mathrm{~dB} / \mathrm{cm}$ will be appear at this dopant concentration, but it can be neglected for device length of about $10 \mu \mathrm{m}$ [5].

Band diagrams of the proposed switch based on the designed parameters calculated in the previous Section are illustrated in FIG. 3. It shows how the changes in refractive index of polymer in the slot shift the band diagrams of even and odd modes. A small decrease in refractive index causes a large change in even mode's and a very small change in odd mode's wavevector at the normalized frequency of $a / \lambda=0.250$.

The coupling length $L_{c}=\pi /\left|k_{\text {even }}-k_{\text {odd }}\right|$ can be calculated directly from FIG. 3. Calculation shows that the switching length vary from $L_{c}=25 a \approx 10 \mu \mathrm{m}$ to $L_{c}=7 a \approx 2.8 \mu \mathrm{m}$ at the normalized frequency of $a / \lambda=0.250$, for $\Delta n_{p o l}=-0.004$ 


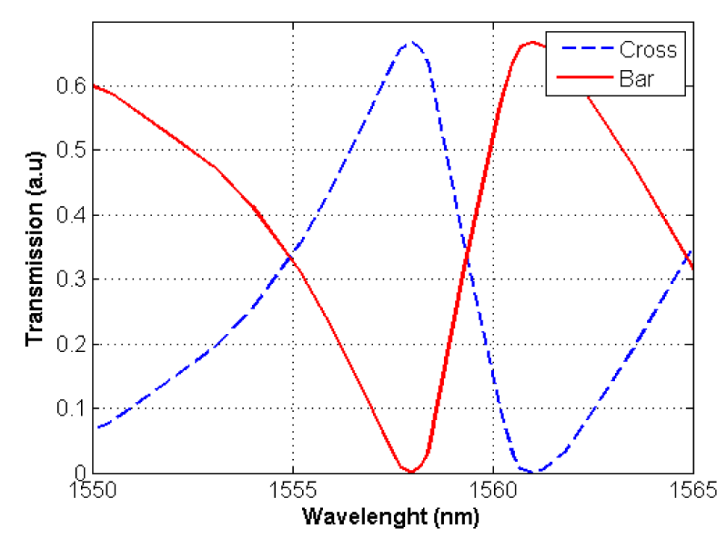

FIG. 4. Transmission specrtum of the proposed switch for bar (solid line) and cross (dashed line).

change of the refractive index of the polymer in the slot. The coupling length is almost the same as that of the thermo-optic photonic crystal switch proposed by Krauss et al. [25], but thermo-optic devices suffer from low speed limited to $1 \mu \mathrm{s}$ [2]. Higher switching speed in Electro-optic devices is one of the advantages of our proposed design. The change in the polymer refractive index can be attained by applying an electrical voltage of $1.52 \mathrm{~V}$ to the electrodes. This voltage is lower than the minimum reported voltage to inject free carrier to the electro-optical switch of coupled PCW [2]. Meanwhile the free carrier injection will contribute to an optical absorption of $14 \mathrm{~dB} / \mathrm{cm}$ [2].

Transmission spectra of the switch are illustrated in FIG. 4 utilizing FDTD method. The power initially transfers through bar ports. A change in refractive index of the structure (which is done by applying voltage to the electrodes), shifts the spectrum, while remaining their shapes $[2,24,25]$, causes the structure switches from bar to cross port at the operation wavelength of $1558 \mathrm{~nm}$ (corresponding to $a / \lambda=0.2503$ for $a=390 \mathrm{~nm}$ ).

\section{TEMPERATURE STABILITY}

Dispersion properties of photonic crystal directional coupler are sensitive to refractive index of the material used in the structure as depicted in FIG. 3. Hence any small change in material refractive index of the photonic crystal directional coupler shifts the dispersion diagram vertically and causes a big change in wavevector of the even mode which causes $\Delta k$ variation in the desired operation frequency [1, 9, and 24]. Meanwhile when the slow light phenomenon is used to improve the light-matter interaction, thermo-optic effect will be enhanced $[9,13]$. Krauss et al. showed that temperature changes of $\Delta \mathrm{T}=38^{\circ} \mathrm{C}$ can create refractive index change of up to $\Delta n=6.8 \times 10^{-3}$ to enable switching in a silicon photonic crystal switch [24, 25]. Hence an accurate temperature control should be considered to guarantee the stable performance of the device for a specified temperature

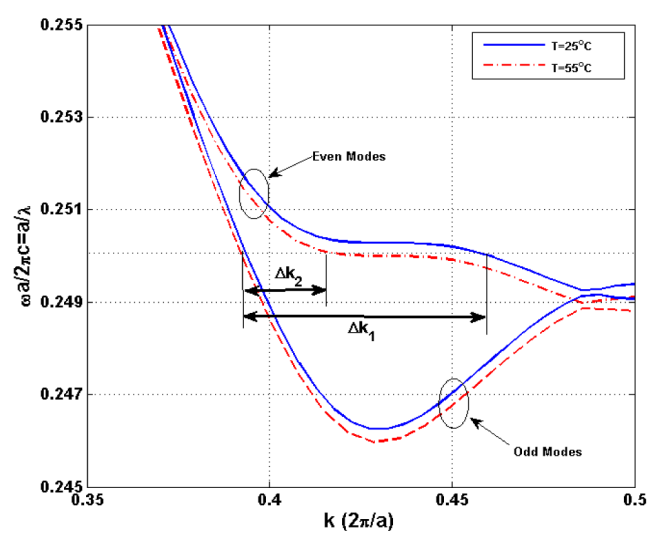

FIG. 5. Band diagram of SPCDC at $\mathrm{T}=25^{\circ} \mathrm{C}$ and $\mathrm{T}=55^{\circ} \mathrm{C}$ which show how temperature variation makes big change in $\Delta k$, which results in the variation of coupling length.

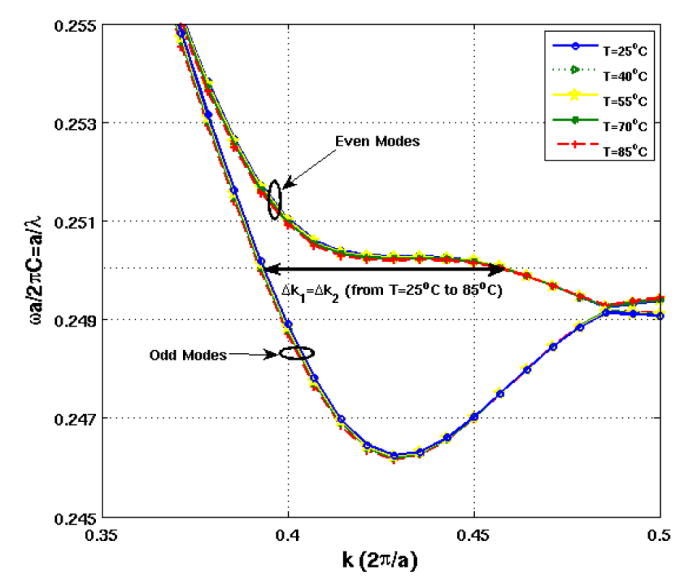

FIG. 6. Band diagram of the proposed switch structure at room temperature of $\mathrm{T}=25^{\circ} \mathrm{C}$ to $\mathrm{T}=85^{\circ} \mathrm{C}$. Performance of the switch is stable for this wide temperature range due to compensation of the silicon's Thermo-Optic coefficient by that of the Polymer's.

range $[1,30]$. The importance of the temperature control in the SPCDC is illustrated in FIG. 5. It shows the changes of the dispersion diagram of the even and odd modes of the proposed structure of FIG. 1 for two different temperatures. The dispersion diagram at room temperature is depicted by a solid line, while the dispersion diagram of the same structure with the same holes and slots filled by polymer, without using negative thermo-optic coefficient of polymer at $\mathrm{T}=55^{\circ} \mathrm{C}$ is depicted by dashed line. It shows a large decrement of $\Delta k$ with temperature. Decrement of $\Delta k$ increases the coupling length; hence, the switch will lose its functionality with temperature variation.

New compensation method for temperature sensitivity introduced by Chung et al. [34] and Lee et al. analyzed a concept to control temperature dependence of the properties of slotted ring resonator devices at a given operating point [33]. Different sign of thermo-optic coefficients of the silicon and the polymer can compensate the refractive index variation 
and prevent undesired change in the switch behavior by temperature [27]. We have utilized this concept to achieve temperature stabilization of group index in silicon slotted photonic crystal waveguides [30]. Here we show our proposed structure will be stable over temperature range of $25^{\circ} \mathrm{C}$ to $85^{\circ} \mathrm{C}$. Thermo-optic coefficients of silicon and polymer have been assumed to be $1.8 \times 10^{-4} /^{\circ} \mathrm{C}$ [25]. Negative thermooptic coefficients of different polymer materials are in the range of $-1 \times 10^{-4} /{ }^{\circ} \mathrm{C}$ to $-4 \times 10^{-4} /{ }^{\circ} \mathrm{C}[36,37]$. Here we have assumed that the thermo-optic coefficient of the proposed polymer is equal to $-2.5 \times 10^{-4} /{ }^{\circ} \mathrm{C}$. Even and odd modes' dispersion diagrams are reproduced in the FIG. 6 for temperature of $25^{\circ} \mathrm{C}$ and $85^{\circ} \mathrm{C}$, indicating a good temperature stability of the switch in this temperature range.

\section{CONCLUSION}

A new design concept of the temperature independent electro-optic switch based on silicon photonic crystal directional coupler (SPCDC) has been introduced. We have maximized the slow light effect in the device by engineering the dispersion properties of the structure to shorten the switching length. Dispersion engineering of the photonic crystal directional coupler has been attained by changing the radii of the innermost rows of the holes in SPCDC and injecting polymer in the slot. Optimum values of the structure parameters have been obtained. Compact length, higher switching speed compared to those of the thermo-optic switches, low activation voltage, easy fabrication and thermal independent switching lengths are the main advantages of the proposed switch. Accurate design and thermal compensation of the polymer makes the performance of the proposed structure stable in the temperature range of $60^{\circ} \mathrm{C}$.

\section{REFERENCES}

1. L. O'Faolain, D. M. Beggs, T. P. White, T. Kampfrath, K. Kuipers, and T. F. Krauss, "Compact optical switches and modulators based on dispersion engineered photonic crystals," IEEE Photon. J. 2, 404-414 (2010).

2. M. J. Zablocki, A. Sharkewy, O. Ebil, S. Shi, and D. Parther, "Electro-optically switched compact coupled photonic crystal waveguide directional coupler," Appl. Phys. Lett. 96, 081110-1 081110-3 (2010).

3. S. P. Anderson, M. Haurylau, J. Zhang, and P. M. Fauchet, "Hybrid photonic crystal microcavity switches on SOI," Proc. SPIE 6477, 647712-1 647712-8 (2007).

4. V. R. Almeida, C. A. Barrios, R. R. Panepucci, and M. Lipson, "All-optical control of light on a silicon chip," Nature 431, 1081-1084 (2004).

5. J. H. Wülbern, A. Petrov, and M. Eich, "Electro-optical modulator in a polymer infiltrated silicon slotted photonic crystal waveguide heterostructure resonator," Opt. Express 17, 304-313 (2009).

6. J. H. Wulbern, J. Hampe, A. Petrove, M. Eich, J. Luo, A.
K. Y. Jen, A. D. Falco, T. F. Krauss, and J. Bruns, "Electro-optic modulation in slotted resonant photonic crystal hetrostructures," Appl. Phys. Lett. 94, 241107-1 241107-3 (2009).

7. T. Baba, "Slow light in photonic crystals," Nature 2, 465-473 (2008).

8. J. K. Hong and S. S. Lee, "Silica-based MMI-MZI thermooptic switch with large tolerance and low PDL," J. Opt. Soc. Korea 9, 119-122 (2005).

9. T. F. Krauss, "Slow light in photonic crystal waveguides," J. Phys. D: Appl. Phys. 40, 2666-2670 (2007).

10. T. Baba and D. Mori, "Slow light engineering in photonic crystals," J. Phys. D: Appl. Phys. 40, 2659-2665 (2007).

11. T. F. Krauss, "Why do we need slow light," Nature Photon. 2, 448-450 (2008).

12. M. Soljacic, S. G. Johnson, S. Fan, M. Ibanescu, E. Ippen, and J. D. Joannopoulos, "Photonic-crystal slow-light enhancement of nonlinear phase sensitivity," J. Opt. Soc. Am. B 19, 2052-2059 (2002).

13. M. Soljacic and J. D. Joannopoulos, "Enhancement of nonlinear effects using photonic crystals," Nature Materials 3, 211-219 (2004).

14. A. Y. Petrov and M. Eich, "Zero dispersion at small group velocities in photonic crystal waveguides," Appl. Phys. Lett. 85, 4866-4868 (2004).

15. L. Juntao, T. P.White, L. O'Faolain, A. Gomez-Iglesias, and T. F. Krauss, "Systematic design of flat band slow light in photonic crystal waveguides," Opt. Express 16, 6227-6232 (2008).

16. J. M. Brosi, C. Koos, L. C. Andreani, M. Waldow, J. Leuthold, and W. Freude, "High-speed low-voltage electrooptic modulator with a polymer-infiltrated silicon photonic crystal waveguide," Opt. Express 16, 4177-4191 (2008).

17. T. Baehr-Jones, B. Penkov, J. Huang, P. Sullivan, J. Davies, J. Takayesu, J. Luo, T. D. Kim, L. Dalton, and A. Jen, "Nonlinear polymer-clad silicon slot waveguide modulator with a half wave voltage of $0.25 \mathrm{~V}$," Appl. Phys. Lett. 92, 92-94 (2008).

18. S. Huang, T. D. Kim, J. Luo, S. K. Hau, Z. Shi, X. H. Zhou, H. L. Yip, and A. K.-Y. Jen, "Slow light enhanced E-O polymer nano-photonic modulator with ultra-high effective in-device r33," Appl. Phys. Lett. 96, 243311 (2010).

19. J. Wu, Y. Li, C. Peng, and Z. Wang, "Numerical demonstration of slow light tuning in slotted photonic crystal waveguide using microfluidic infiltration," Opt. Commun. 284, 2149-2152 (2011).

20. R. Ding, T. Baehr-Jones, W.-J. Kim, A. Spott, M. Fournier, J.-M. Fedeli, S. Huang, J. Luo, A. K.-Y. Jen, L. Dalton, and M. Hochberg, "Sub-volt silicon-organic electrooptic modulator with $500 \mathrm{MHz}$ bandwidth," J. Lightwave Technol. 29, 1112-1117 (2011).

21. X. Wang, C. Lin, S. Chakravarty, J. Luo, A. K. Y. Jen, and R. T. Chen, "Effective in-device $\mathrm{r} 33$ of $735 \mathrm{pm} / \mathrm{V}$ on electro-optic polymer infiltrated silicon photonic crystal slot waveguides," Opt. Lett. 36, 882-884 (2011).

22. S. Huang, J. Luo, H. Yip, A. Ayazi, X. H. Zhou, M. Gould, A. Chen, T. Baehr-Jones, M. Hochberg, and A. K.-Y. Jen, "Efficient poling of electro-optic polymers in thin films and silicon slot waveguides by detachable pyroelectric crystals," Adv. Mater. 24, OP42-OP47 (2012). 
23. W. C. Kim and D. W. Park, "Analysis of temperature effects on Raman silicon photonic devices," J. Opt. Soc. Korea 12, 288-297 (2008).

24. D. M. Beggs, T. P. White, L. O'Faolain, and T. F. Krauss, "Ultra compact and low-power optical switch based on silicon photonic crystals," Opt. Lett. 33, 147-149 (2008).

25. D. M. Beggs, T. P. White, L. Cairns, L. O'Faolain, and T. F. Krauss, "Ultrashort photonic crystal optical switch actuated by microheater," IEEE Photon. Technol. Lett. 21, 24-26 (2009).

26. B. A. Rose, A. J. Maker, and A. M. Armani, "Characterization of thermo-optic coefficient and material loss of high refractive index silica sol-gel films in the visible and near-IR," Opt. Material Express 2, 671-681 (2012).

27. C. Karnutsch, C. L. C. Smith, A. Graham, S. TomljenovicHanic, R. C. McPhedran, B. J. Eggleton, L. O'Faolain, T. F. Krauss, S. Xiao, and N. A. Mortensen, "Temperature stabilization of optofluidic photonic crystal cavities," Appl. Phys. Lett. 94, 231114-1 231114-3 (2009).

28. S. Haishan, A. Szep, S. Shouyuan, D. Prather, L. Zhou, R. S. Kim, and D. Abeysinghe, "Achieving higher modulation efficiency in electrooptic polymer modulator with slotted silicon waveguide," J. Lightwave Technol. 29, 3310-3318 (2011).

29. C. Changming, S. Xiaoqiang, W. Fei, Z. Feng, W. Hui, S. Zuosen, C. Zhanchen, and Z. Daming, "Electro-optic modulator based on novel organic-inorganic hybrid nonlinear optical materials," IEEE J. Quantum Electron. 48, 61-66 (2012).

30. H. Aghababaeian, M. H. Vadjed-Sameie, and N. Granpayeh, "Temperature stabilization of group index in silicon slotted photonic crystal waveguides,” J. Opt. Soc. Korea 15, 398-402 (2011).
31. M. Ebnali-Heidari, C. Grillet, C. Monat, and B. J. Eggleton, "Dispersion engineering of slow light photonic crystal waveguides using microfluidic infiltration," Opt. Express 17, 1628-1635 (2009).

32. http://ab-initio.mit.edu/wiki/index.php/MIT_Photonic_Bands.

33. J. M. Lee, D. J. Kim, G. H. Kim, O. K. Kwon, K. J. Kim, and G. Kim, "Controlling temperature dependence of silicon waveguide using slot structure," Opt. Express 16, 1645-1652 (2008).

34. Y. Chung, J. Song, W. Han, and U. Paek, "New compensation method for temperature sensitivity of fiber Brags grating using bi-metal," J. Opt. Soc. Korea 7, 84-88 (2003).

35. S. M. Lee, "Passive temperature compensating package for optical long period fiber grating," J. Opt. Soc. Korea 3, 74-79 (1999).

36. D. Lee, K. H. Kim, S. H. Hwang, M. H. Lee, and E. H. Lee, "Optimization of thermo-optic parameters for temperatureinsensitive LPWG refractometers," ETRI Journal 28, 739-744 (2006).

37. J. Teng, P. Dumon, W. Bogaerts, H. Zhang, X. Jian, X. Han, M. Zhao, G. Morthier, and R. Baets, "Athermal siliconon-insulator ring resonators by overlaying a polymer cladding on narrowed waveguides," Opt. Express 17, 1462714633 (2009).

38. C. Park, K. Joo, S. W. Kang, and H. R. Kim, "A PDMScoated optical fiber Bragg grating sensor for enhancing temperature sensitivity,” J. Opt. Soc. Korea 15, 329-334 (2011).

39. J. Hou, D. Gao, H. Wu, R. Hao, and Z. Zhou, "Flat band slow light in symmetric line defect photonic crystal waveguides," IEEE Photon. Technol. Lett. 21, 1571-1573 (2009). 